\title{
Visual discrimination between two sexually deceptive Ophrys species by a bee pollinator
}

\author{
M. Streinzer $\cdot$ T. Ellis $\cdot$ H. F. Paulus $\cdot$ \\ J. Spaethe
}

Received: 30 December 2009/ Accepted: 21 April 2010/Published online: 22 May 2010

(C) The Author(s) 2010. This article is published with open access at Springerlink.com

\begin{abstract}
Almost all species of the orchid genus Ophrys are pollinated by sexual deception. The orchids mimic the sex pheromone of receptive female insects, mainly hymenopterans, in order to attract males seeking to copulate. Most Ophrys species have achromatic flowers, but some exhibit a coloured perianth and a bright, conspicuous labellum pattern. We recently showed that the pink perianth of Ophrys heldreichii flowers increases detectability by its pollinator, males of the long-horned bee Eucera berlandi. Here we tested the hypothesis that the bright, complex labellum pattern mimics the female of the pollinator to increase attractiveness toward males. In a dualchoice test we offered $E$. berlandi males an $O$. heldreichii flower and a flower from $O$. dictynnae, which also exhibits a pinkish perianth but no conspicuous labellum pattern. Both flowers were housed in UV-transmitting acrylic glass boxes to exclude olfactory signals. Males significantly preferred $O$. heldreichii to $O$. dictynnae flowers. In a second experiment, we replaced the perianth of both flowers with identical artificial perianths made from pink card, so that only the labellum differed between the two flower stimuli. Males then chose between both stimuli at random, suggesting that the presence of a labellum pattern does not affect their choice. Spectral measurements revealed higher colour contrast with the background of the perianth of
\end{abstract}

Handling Editor: Lars Chittka.

M. Streinzer $(\bowtie) \cdot$ T. Ellis · H. F. Paulus · J. Spaethe Department of Evolutionary Biology, Faculty of Life Sciences, University of Vienna, Althanstraße 14, 1090 Vienna, Austria e-mail: martin.streinzer@univie.ac.at

T. Ellis

Faculty of Life Sciences, University of Manchester, Manchester, UK
O. heldreichii compared to O. dictynnae, but no difference in green receptor-specific contrast or brightness. Our results show that male choice is guided by the chromatic contrast of the perianth during the initial flower approach but is not affected by the presence of a labellum pattern. Instead, we hypothesise that the labellum pattern is involved in aversive learning during post-copulatory behaviour and used by the orchid as a strategy to increase outcrossing.

Keywords Ophrys heldreichii · Colour vision · Sexual deception - Signal evolution - Object detection . Pollination

\section{Introduction}

Long before the discovery of sexual deception as pollination syndrome, some authors had already recognised the apparent visual resemblance between flowers of the orchid genus Ophrys and insect bodies (Linnaeus 1745; Darwin 1862). One early hypothesis, for instance, suggested that the orchids mimic a feeding insect to advertise a potential food source to conspecifics of the species being imitated (Darwin 1862 and citations therein). From the 1920s on, when Pouyanne discovered that the orchids in fact achieve pollination by means of sexual deception of male insects, the insect-like appearance of the labellum was regarded as imitating various female signals, such as the body shape, wings, or abdominal markings (Correvon and Pouyanne 1916; Kullenberg 1961; Paulus and Gack 1990; Paulus 2006, 2007).

Nearly 100 years later, pollination by sexual deception has been described from all continents except Antarctica. The syndrome has so far only been discovered in the orchid family (Orchidaceae) and has evolved several times 
independently (van der Pijl and Dodson 1966; Nilsson 1992). The orchids mimic species-specific mating signals in order to attract male insects, especially bees and other hymenopterans, and elicit courtship behaviour. Whilst attempting to copulate with the flower's labellum, the males come into contact with the sticky viscidia and the pollinaria become attached to their bodies. Should the male subsequently be deceived by another orchid, this pollen is transferred to the stigma of the next flower and pollination occurs (Kullenberg 1961; Paulus and Gack 1990; Ciotek et al. 2006).

Attraction of the target-male can only be successful if the orchid mimics the key signals deployed in insect mating communication. In hymenopterans, the major communication channel is olfaction. Virgin females produce a sex pheromone that usually consists of a blend of semiochemicals that signals mating readiness (Ayasse et al. 2001; Paxton 2005). The olfactory communication is highly specific and thus constitutes a private communication channel, i.e. only males of the target species can decipher the message sent by the female (Endler 1993; Ayasse et al. 2001; Schaefer et al. 2004). The chemical profile of the flower closely resembles the behaviourally active substances of the model insect; as a consequence, pollinator attraction in sexually deceptive orchids is highly specific (Schiestl et al. 1999; Ayasse et al. 2000; Schiestl 2005; Mant et al. 2005).

The Mediterranean genus Ophrys comprises about 260 species of which almost all are sexually deceptive and usually attract males of only one species (Paulus and Gack 1990; Ayasse et al. 2000). Due to the highly specific olfactory attraction, visual signals are assumed to play only a minor role (Kullenberg 1961; Kullenberg and Bergström 1976). Most Ophrys flowers are indeed dull (Delforge 2006), probably to avoid visual attraction of unspecific visitors and thus the risk of pollen loss. However, some Ophrys species exhibit brightly-coloured sepals and petals besides the labellum (henceforth referred to as perianth for simplicity). Recent investigations have shown that such potential visual signals can increase detectability and attractivity to the pollinator on the one hand, but may also impose additional costs (Spaethe et al. 2007, 2010; Streinzer et al. 2009). Moreover, some Ophrys species show a bright and conspicuous pattern on the labellum which has been hypothesised to mimic traits of the pollinator's female, such as the wings or body-markings to make the flower more attractive for the males (Kullenberg 1961; Paulus 2006, 2007). However, the labellum pattern is relatively small and the compound eyes of hymenopterans allow only a coarse spatial resolution (Land 1997) and thus it is not clear whether males can indeed perceive these patterns and mistake them for female traits.

Here we tested the hypothesis that the conspicuous labellum pattern mimics behaviourally-relevant parts of the

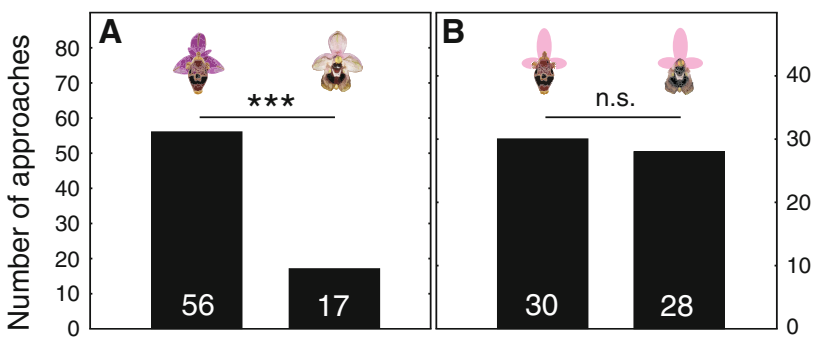

Fig. 1 Male choices for a intact flowers of Ophrys heldreichii (left column) and O. dictynnae (right column) (73 approaches in total), and for $\mathbf{b}$ flowers where the original perianth was replaced by an artificial one made from pink card (58 approaches). Number of approaches are given within the column. ${ }^{* * *} P<0.001$, n.s., $P>0.05$; $\chi^{2}$-test

female's body and thus increase attractiveness to males. We conducted dual-choice experiments with two Ophrys species that both are pollinated by long-horned bees. Ophrys heldreichii, which is pollinated by Eucera berlandi, possesses a bright and complex labellum pattern, while O. dictynnae lacks such a conspicuous pattern (Figs. 1, 2). Both species have a pink perianth, although they differ slightly with respect to the colour for a human observer. We presented both flower types in UV-transmitting acrylic glass boxes to the pollinator of $O$. heldreichii, Eucera berlandi. If the proposed hypothesis is correct, we expect that males prefer flowers with the conspicuous labellum pattern over flowers without such a pattern.

\section{Materials and methods}

Study sites and species

Experiments were carried out on Crete, about $5 \mathrm{~km}$ northeast of Neapolis (N 35 $15^{\prime} 13^{\prime \prime}$, E $25^{\circ} 38^{\prime} 03^{\prime \prime}$ ) with Heldreich's bee orchid, Ophrys heldreichii SCHLECHTER (Orchidaceae), which is member of the $O$. oestrifera group, and its specific pollinator, males of the long-horned bee Eucera (Synhalonia) berlandi DUSMET (Apoidea, Apidae, Eucerini). As second orchid species, Ophrys dictynnae DELFORGE, a recently described representative of the $O$. tenthredinifera group, was chosen for the discrimination experiments. This orchid differs from $O$. heldreichii in the absence of a conspicuous labellum pattern and in perianth colour, which appears slightly brighter to a human observer (Figs. 1, 2). O. dictynnae is also pollinated by males of a long-horned bee species, Eucera nigrita FRIESE (Streinzer and Paulus, unpublished). For the experiments, intact flowers were collected from various sites within an area of about $40 \mathrm{~km}$ around the experimental site. O. heldreichii and $O$. dictynnae do not occur at the experimental site and thus all tested E. berlandi males were naïve to both species. 
Behavioural experiments

To test whether the males discriminate between the two orchid species we conducted a dual-choice experiment. Two flowers, one of each species, were presented simultaneously to individual males of $E$. berlandi and choices were recorded. To eliminate odour-induced bias the flowers were presented in UV-transmitting acrylic glass boxes at a height of approx. $30 \mathrm{~cm}$ and $10 \mathrm{~cm}$ apart from each other (Spaethe et al. 2007). Since visual stimuli alone are not sufficient to attract pollinators, we placed 20-25 flowers of $O$. heldreichii in a 2-1 acrylic jar. Air was channelled through the jar by means of an aquarium pump (SCHEGO optimal, Offenbach am Main, Germany) at a rate of $250 \mathrm{l} /$ h. The odour-saturated outflow was then distributed equally to both acrylic glass boxes via a Y-tube (Spaethe et al. 2007). The flowers were presented in front of a piece of green leaf of a sea onion (Urginea maritima).

In the first experiment we presented original flowers of both species to test whether males discriminate between them. In a second experiment, to separate the role of the perianth and the labellum, we removed the original sepals and petals in both flowers and replaced them with an identical artificial perianth cut from coloured card that approximately resembled the original perianth of $O$. heldreichii in colour, size and shape (Fig. 3, Streinzer et al. 2009). In this test, the only difference between both test flowers was the labellum.

All approaches of individual males that resulted in contact with one of the acrylic glass boxes were counted as a decision. Males were then immediately caught and marked with a coloured marker on the thorax or abdomen. Repeat visits by individual males were excluded from the analysis. The position of the flowers was interchanged after every fifth visit to exclude any bias due to side. Moreover, the orchid flowers were replaced every couple of hours from a random sample of flowers collected from different populations (see below).

\section{Flower morphology}

Flowers from both species differ slightly in shape and size. To account for size effects on choice behaviour we measured sepal and labellum surface of $O$. heldreichii and $O$. dictynnae flowers. Specimens were collected from the same populations where we took the flowers for the behavioural experiments (one population of $O$. heldreichii, two of $O$. dictynnae). We randomly chose one flower from each plant $\left(\mathrm{N}_{O}\right.$. heldreichii $\left.=15, \mathrm{~N}_{O \text {. dictynnae }}=27\right)$ for morphological and spectral measurements.

The sepals were separated from the labellum and all parts were transferred onto a piece of adhesive tape together with a size reference, and photographed with a digital camera (Nikon D70s, AF 105 mm/2,8 D; Nikon, Japan). Pictures were subsequently transferred to a personal computer and analyzed with ImageJ (http://rsbweb.nih.gov/ij/). We measured the total area of the three sepals and of the labellum (without the stylum).

Spectral measurements and bee colour space calculations

Spectral reflectance measurements of the perianth were carried out on the same flowers as for morphological measurements. Spectra were obtained by means of a USB 2000 spectrometer with a balanced deuterium/halogen light source (Ocean Optics B.V., Duiven, The Netherlands) between 300 and $700 \mathrm{~nm}$ on an approx. $0.25 \mathrm{~cm}^{2}$ area of the median sepal. The spectrometer was calibrated with a white PTFE standard (Diffuse Reflectance Standard, WS-1, Ocean Optics). Spectral data were processed in Microsoft Excel 2002.

To estimate the bee specific receptor contrasts and perceptual colour distance between the perianth and the stimulus background we applied the bee colour hexagon (Chittka 1992). Since the receptor sensitivities of $E$. berlandi are unknown, we used the spectral sensitivity functions of Apis mellifera (Peitsch et al. 1992). This approach seems appropriate since most bees have been found to share similar receptor properties with respect to number and shape of the sensitivity curves (Peitsch et al. 1992). Colour loci and receptor-specific excitations were calculated using standard procedures (Spaethe et al. 2001; Chittka and Kevan 2005). It is assumed that photoreceptors adapt, depending on the ambient illumination, to the background (Chittka 1992). We therefore chose an averaged reflection spectrum of several leaves from different flowers which are abundant in the experimental area (including Urginea maritima) as adaptation background (Fig. 3a). Colour distance between colour loci of the flower perianths and the stimulus background were calculated as the Euclidean distance in the colour hexagon. Green receptor-specific contrast was calculated as the absolute value of the difference between the calculated excitation of the long-wavelength receptor by the background and the flower. Brightness was calculated as the sum of all three receptor excitations (Spaethe et al. 2001).

\section{Statistics}

All statistical analyses were performed using SPSS 11.5 (SPSS Inc., Chicago, IL). To test if behavioural responses of the males differ significantly from chance a $\chi^{2}$-test was applied. For morphological measurements and colour contrast we first validated whether the two populations of $O$. dictynnae differ from each other. All measured 
parameters were found to be not significantly different and thus data from both populations were pooled (data not shown). To test whether spectral and morphological parameters differ between the two orchid species we applied a Mann-Whitney $U$-test. All $P$-values above 0.05 were considered as statistically non-significant.

\section{Results}

\section{Behavioural experiments}

When males of the long-horned bee E. berlandi were allowed to choose between intact flowers of $O$. heldreichii and $O$. dictynnae, they significantly preferred $O$. heldreichii over $O$. dictynnae $\left(\chi^{2}=20.84, P<0.001, N=73\right.$; Fig. 1a). Since the olfactory signal was the same for both stimuli, the result clearly shows that males use visual cues or signals to discriminate between the two species of orchids. To test whether the preference of $O$. heldreichi flowers is due to differences of the perianth and/or the labellum, we replaced the original perianth with an artificial one, which was identical for both flower stimuli. We found that when only the labellum differs, males do not show a preference for either of the two flower types $\left(\chi^{2}=0.07, P=0.79, N=58\right.$; Fig. $\left.1 \mathrm{~b}\right)$.

Flower morphology measurements

Perianth surface did not differ between both orchid species $\left(Z=0.25, P=0.80 ; \mathrm{N}_{O}\right.$. heldreichii $=15, \mathrm{~N}_{O \text {. dictynnae }}=$ 27; Fig. 2a). When we compared the labellae, O. dictynnae showed a significantly larger labellum than $O$. heldreichii $\left(Z=-4.19, P<0.001 ; \mathrm{N}_{O}\right.$. heldreichii $=15, \mathrm{~N}_{O \text {. dictynnae }}=$ 27; Fig. 2b). However, the behavioural data indicate that

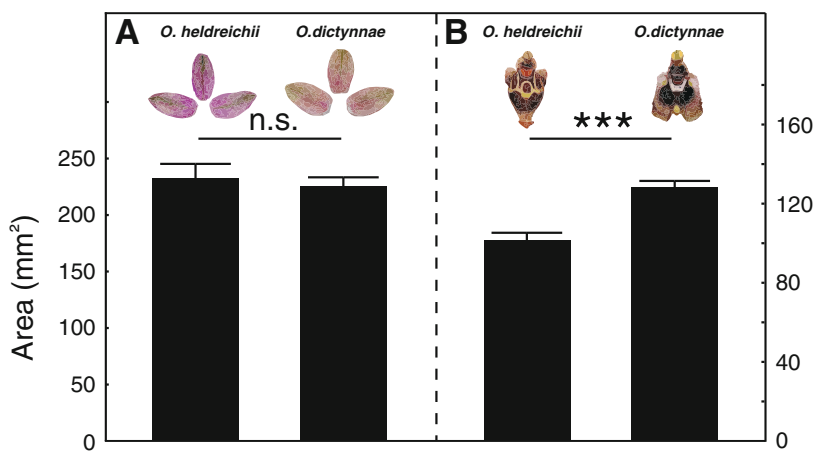

Fig. 2 Flower morphology of the perianth (a) and the labellum (b) of $O$. heldreichii (left column) and $O$. dictynnae (right column). Perianth size does not differ between the two species. However, O. dictynnae has a significantly larger labellum compared to $O$. heldreichii. $\mathrm{N}_{O}$. heldreichii $=15, \mathrm{~N}_{O}$. dictynnae $=27$; means $\pm \mathrm{SEM}$; *** $P<0.001$, n.s., $P>0.05$; Mann-Whitney $U$-test neither labellum size nor the presence/absence of a labellum pattern affect male choice.

Spectral measurements

The spectral reflectance curves of the $O$. heldreichii and O. dictynnae sepals show a different shape (Fig. 3a-c). To examine whether the two species also differ in their appearance to the bee's visual system we plotted the perianth colours in a bee specific colour space (Chittka 1992; Fig. 3d; Table 1). The colour loci of the two populations of $O$. dictynnae were found to overlap and were therefore pooled for further analysis (data not shown). $O$. heldreichii flowers exhibit a higher colour contrast to the background compared to $O$. dictynnae $(Z=4.55$, $P<0.001)$. Beside the difference in colour contrast, the two species also differ in hue, i.e. the angle they subtend from the centre $\left(\alpha_{\text {heldreichii }}=100.6 \pm 10.9^{\circ} ; \alpha_{\text {dictynnae }}=\right.$ $124.8 \pm 22.2^{\circ} ;$ Mean \pm SD; Fig. $3 \mathrm{~d} ; \quad Z=-3.54, \quad P<$ 0.001 ; where $0^{\circ}$ corresponds to the vector between centre and the UV corner of the colour hexagon). Green receptor contrast, a sensory channel used by bees to detect objects at small angular sizes, and total brightness did not differ between the two species $(Z=-0.46, P=0.65$ and $Z=1.38, P=0.17$, respectively; Table 1$)$.

\section{Discussion}

In this study we tested whether $E$. berlandi males use visual cues to discriminate between flowers of two species of the sexually deceptive orchid genus Ophrys. We chose two species that differ both in the appearance of the labellum and also the colour of the perianth. In dual-choice experiments we found that males are clearly able to discriminate between both species visually. However, when we replaced the coloured perianth with an artificial one, males chose both flower types at random. From these results we can conclude that (1) the labellum pattern of $O$. heldreichii is not involved in male attraction and that (2) when males can choose between flowers they prefer flowers with a perianth with the higher chromatic contrast.

Various types of labellum patterns are present in the genus Ophrys and were assumed to mimic female traits to increase attractiveness to the males (Paulus 1988, 2006, 2007). However, whether the males indeed mistake these patterns as females or at least key parts of a female's body, has not yet been shown. O. heldreichii exhibits a whitish, highly contrasting pattern on a dark labellum, which is also found of similar type in all species of the Ophrys oestrifera group (Delforge 2006). At a first glance the labellum pattern shows no similarity to the female of the pollinator species, which is reddish-brownish and without any conspicuous 


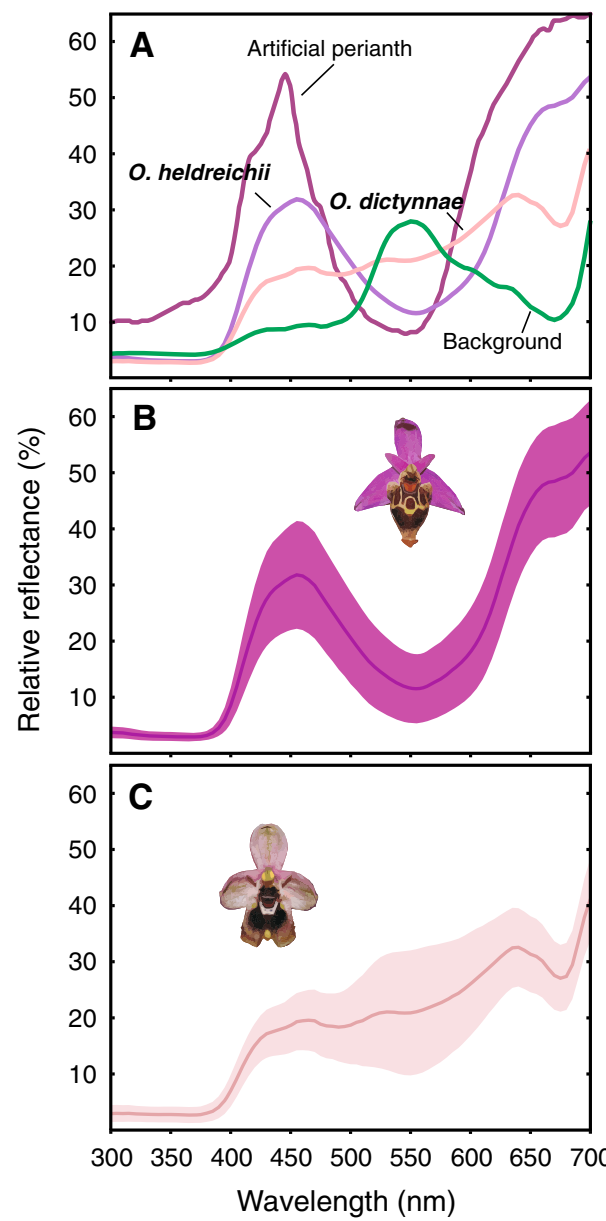

Fig. 3 a-c Spectral reflectance curves of the perianth of Ophrys heldreichii (dark violet), Ophrys dictynnae (pale pink), the artificial perianth (dark pink) and the background (green) as a function of wavelength. In a only mean reflectance of the flower perianth are given $\left(\mathrm{N}_{O}\right.$. heldreichii $=15, \mathrm{~N}_{O}$. dictynnae $=27, \mathrm{~N}_{\text {Background }}=5$, see text). b and c show the mean reflectance of $O$. heldreichii and $O$. dictynnae, respectively, presented with the standard deviation of the mean to visualize the variation within the species. $d$ The colour loci of Ophrys heldreichii (dark violet), O. dictynnae (light pink), the artificial perianth and two major food plants (Vicia cracca and Salvia fructicosa) represented in the colour hexagon model (Chittka 1992). The large circle denotes 0.1 hexagon units around the centre. All loci within this circle are assumed to appear achromatic to the bee (Chittka and Kevan 2005). The grey line indicates the loci of pure spectral lights at background intensity. Colour distances in the hexagon space are calculated as the Euclidean distance between two loci (Chittka 1992)

Table 1 Chromatic contrast, green contrast and brightness in relation to the background

\begin{tabular}{llll}
\hline & Ophrys heldreichii $(n=15)$ & Ophrys dictynnae $(n=27)$ & Mann-Whitney $U$-test \\
\hline Chromatic contrast (Hexagon units) & $0.23 \pm 0.03$ & $0.16 \pm 0.04$ & $P<0.001$ \\
Green contrast (dimensionless) & $0.09 \pm 0.05$ & $0.09 \pm 0.05$ & NS \\
Brightness (dimensionless) & $1.74 \pm 0.21$ & $1.62 \pm 0.25$ & NS \\
\hline
\end{tabular}

The values represent means \pm standard deviations. Values were calculated using the colour hexagon model (Chittka 1992). For the calculation we used the spectral sensitivity curves of Apis mellifera (Peitsch et al. 1992). Note that for the green-receptor contrast the absolute values are given (Spaethe et al. 2001)

bright patterns on the body or wings. Our results show that the presence of such a pattern does not affect a male's initial flower choice, and thus it is most likely that the pattern does not mimic any female traits that increase attractivity to the males. What then is the functional significance of the labellum pattern? We speculate that it may be involved in aversive learning during post-copulating behaviour, that is, the male associates the unsuccessful copulation attempt 
with the labellum pattern and avoids further visits to flowers with an identical pattern (see Paulus 1988). Two observations support this hypothesis. First, males of E. berlandi do not fly away immediately after pseudocopulation but hover a few centimetres in front of the flower for periods of several seconds up to a minute (personal observation). Although the compound eyes limit the spatial resolution of diminutive and fine patterns, at this short distance the males might be able to resolve the labellum pattern (Lunau et al. 2009). Recent investigations have shown that bees are capable of learning and discriminating even very complex and fine-scale natural scenes (Dyer et al. 2008b). Second, pattern size and shape are found to be highly variable among flowers of a population, but very similar among flowers within a plant (Paulus 2007, personal observation). The high variation of this floral trait among $O$. heldreichii individuals might facilitate increased outcrossing, since, if the males are indeed able to learn the pattern, they would avoid other flowers with similar patterns to ones they had recently visited, but would be more willing to visit flowers from another plant with a different pattern. However, this hypothesis requires confirmation from behavioural studies showing that males are able to learn and discriminate patterns from different plants, and that males avoid flowers with patterns similar to those that have been encountered during a previous pseudocopulation.

Although we could clearly show that $E$. berlandi males are not attracted by the labellum pattern of $O$. heldreichii flowers in their initial flower choice, our results cannot simply be generalised for all Ophrys species. The labellum of $O$. speculum, for instance, possesses a conspicuous shiny blue mirror that also reflects in the ultraviolet (UV). In dual-choice tests, males of the sphecid wasp Dasyscolia ciliata, the pollinator of $O$. speculum, preferred flowers with an unmanipulated labellum to flowers whose mirror had been manipulated to reduce the UV reflectance (Paulus 2007, personal observations). However, for most Ophrys species with labellum patterns the functional significance of such markings in flower-pollinator communication is still unknown.

The only visual trait we could find that affects male choice behaviour was the colour of the perianth. The perianths of $O$. heldreichii flowers have a higher colour contrast (but identical green-receptor and brightness contrasts) and are more attractive compared to $O$. dictynnae (but see Vereecken and Schiestl 2009). In a recent study we found that the detection of $O$. heldreichii flowers by E. berlandi from a distance of approximately $30 \mathrm{~cm}$ was improved when the green contrast between the perianth and the background increased, but colour contrast was found to have no effect (Streinzer et al. 2009). However, in the present study, males could choose between the two flower stimuli within a range of a few centimetres which most likely enabled them to use the chromatic channel that only works at large visual angles (Giurfa et al. 1996; Dyer et al. 2008a). Flower choice experiments with naive bumblebees revealed a preference for colours providing a large colour contrast to the background and might reflect a more general preference in the flower choice behaviour of bees (Lunau 1990; Lunau et al. 1996). Besides colour contrast, the spectral purity of a target (i.e. saturation) may also affect colour choice (Lunau et al. 1996). Our experimental setup, however, does not allow to disentangle whether the choice behaviour of E. berlandi males is primarily based on the colour contrast between the perianth and the background or the saturation of the perianth or both. Alternatively, males might prefer the hue of the perianth of $O$. heldreichii flowers compared to that of $O$. dictynnae because it more closely resembles the flower colour of the females' most frequent host plants (e.g. Vicia cracca and Salvia fructico$s a$, see Spaethe et al. 2007 for spectral reflectance curves). However, the colour loci of the food plants overlap at least partially with the loci of both orchids, $O$. heldreichii and O. dictynnae, respectively (Fig. 3d). Additional behavioural experiments are necessary to disentangle the role of colour contrast, saturation and hue in male attraction.

Pollinator visitation rate is a critical factor determining reproductive success in Ophrys (Vandewoestijne et al. 2009). Thus, it is surprising that selection did not lead to a perianth colour that provides higher colour contrast (and is thus more attractive) in $O$. dictynnae, which is also pollinated by a long-horned bee. We suggest two non-mutually exclusive hypotheses which may account for this discrepancy. First, males of the two pollinator species, E. berlandi and $E$. nigrita, may exhibit preferences for different colour hues which resulted in a separation of the perianth colours of the two Ophrys species by natural selection. We observed males and females of $E$. berlandi foraging on various food plants at the experimental site (see above). However, we neither know the colour of the major host plants of E. nigrita, nor if the flowers visited by the two pollinator-species cover different areas in the bees' colour space. Second, differences in colour contrast (and also hue) between the two Ophrys species are caused by differences in the expression of floral pigments in the perianth. Anthocyanins are probably the major pigment in the pink perianths of Ophrys flowers (Arditti and Fisch 1974; Uphoff 1979), and an increased anthocyanin concentration may lead to a higher colour contrast. However, the production of flower pigments most probably imposes costs on the plant and $O$. dictynnae might be more limited in resources that can be allocated to the anthocyanin production compared to $O$. heldreichii. Again, further investigations are necessary to fully understand the ultimate and proximate mechanisms accounting for differences in perianth colour among Ophrys. 
In conclusion, the conspicuous labellum pattern of $O$. heldreichii flowers does not increase pollinator attraction during the initial flower approach flight. Instead, we suggest that the pattern is involved in aversive learning by the males during post-copulatory behaviour and constitute a strategy of the orchid to increase outcrossing. Labellum patterns are commonly found within the Ophrys genus but differ largely in size, colour and complexity, and thus most likely in their function for flower-pollinator interaction. Further behavioural investigations could provide insights into whether a particular pattern-type is associated with a specific pollinator group, or mimic a particular female trait as previously suggested.

Acknowledgments We would like to thank AG Dyer for valuable input on colour measurements. This study was supported by a grant from the Austrian Academy of Sciences (KIÖS) to HFP and from the Austrian Science Fund (FWF, grant No. P21521-B17) to JS.

Open Access This article is distributed under the terms of the Creative Commons Attribution Noncommercial License which permits any noncommercial use, distribution, and reproduction in any medium, provided the original author(s) and source are credited.

\section{References}

Arditti J, Fisch MH (1974) Anthocyanins of the Orchidaceae: distribution, heredity, functions, synthesis, and localization. In: Arditti J (ed) Orchid biology: reviews and perspectives. Cornell University Press, London, pp 117-156

Ayasse M, Schiestl FP, Paulus HF, Löfstedt C, Hansson B, Ibarra F, Francke W (2000) Evolution of reproductive strategies in the sexually deceptive orchid Ophrys sphegodes: how does flowerspecific variation of odor signals influence reproductive success? Evolution 54:1995-2006

Ayasse M, Paxton RJ, Tengö J (2001) Mating behaviour and chemical communication in the order Hymenoptera. Annu Rev Entomol $46: 31-78$

Chittka L (1992) The colour hexagon: a chromaticity diagram based on photoreceptor excitations as a generalized representation of colour opponency. J Comp Physiol A 170:533-543

Chittka L, Kevan PG (2005) Flower colour as advertisement. In: Dafni A, Kevan PG, Husband BC (eds) Practical pollination biology. Enviroquest, Cambridge, pp 157-196

Ciotek L, Giorgis P, Benitez-Vieyra S, Cocucci AA (2006) First confirmed case of pseudocopulation in terrestrial orchids of South America: pollination of Geoblasta pennicillata (Orchidaceae) by Campsomeris bistrimacula (Hymenoptera, Scoliidae). FLORA 201:365-369

Correvon H, Pouyanne A (1916) Un curieux cas de mimétisme chez les Ophrydées. J Soc Nat Hortic Fr 17:29-31

Darwin CR (1862) On the various contrivances by which British and Foreign orchids are fertilized by insects. John Murray \& Sons, London

Delforge P (2006) Orchids of Europe, North Africa and Middle East. Timber Press, Oregon

Dyer AG, Spaethe J, Prack S (2008a) Comparative psychophysics of bumblebee and honeybee colour discrimination and object detection. J Comp Physiol A 194:617-627
Dyer AG, Rosa MGP, Reser DH (2008b) Honeybees can recognise images of complex natural scenes for use as potential landmarks. J Exp Biol 211:1180-1186

Endler JA (1993) Some general comments on the evolution and design of animal communication systems. Philos Trans R Soc Lond B 340:215-225

Giurfa M, Vorobyev M, Kevan P, Menzel R (1996) Detection of coloured stimuli by honeybees: minimum visual angles and receptor specific contrasts. J Comp Physiol A 178:699-709

Kullenberg B (1961) Studies in Ophrys pollination. Zool Bidr Från Upps 34:1-340

Kullenberg B, Bergström G (1976) Hymenoptera Aculeata males as pollinators of Ophrys orchids. Zool Scr 5:13-23

Land MF (1997) Visual acuity in insects. Annu Rev Entomol 42: $147-177$

Linnaeus C (1745) Öländska och Gothländska Resa. Gottfried Kiesewetter, Stockholm

Lunau K (1990) Colour saturation triggers innate reactions to flower signals: flower dummy experiments with bumblebees. J Comp Physiol A 166:827-834

Lunau K, Wacht S, Chittka L (1996) Colour choices of naive bumble bees and their implications for colour perception. J Comp Physiol A 178:477-489

Lunau K, Unseld K, Wolter F (2009) Visual detection of diminutive floral guides in the bumblebee Bombus terrestris and the honeybee Apis mellifera. J Comp Physiol A 195:1121-1130

Mant J, Brändli C, Vereecken NJ, Schulz CM, Francke W, Schiestl FP (2005) Cuticular hydrocarbons as sex pheromone of the bee Colletes cunicularius and the key to its mimicry by the sexually deceptive orchid, Ophrys exaltata. J Chem Ecol 31:1765-1787

Nilsson LA (1992) Orchid pollination biology. TREE 7:255-259

Paulus HF (1988) Co-Evolution und einseitige Anpassungen in Blüten-Bestäubersystemen: Bestäuber als Schrittmacher in der Blütenevolution. Verh Dtsch Zool Ges 81:25-46

Paulus HF (2006) Deceived males-pollination biology of the Mediterranean orchid genus Ophrys (Orchidaceae). J Eur Orchid 38(2):303-353

Paulus HF (2007) Wie Insekten-Männchen von Orchideenblüten getäuscht werden-Bestäubungstricks und Evolution in der mediterranen Ragwurzgattung Ophrys. In: Gusenleitner F (ed) Evolution-Phänomen Leben, vol 20. Denisia, Linz, pp 255-294

Paulus HF, Gack C (1990) Pollinators as prepollinating isolation factors: evolution and speciation in Ophrys (Orchidaceae). Isr $\mathbf{J}$ Bot 39:43-79

Paxton RJ (2005) Male mating behaviour and mating systems of bees: an overview. Apidologie 36:145-156

Peitsch D, Fietz A, Hertel H, de Souza J, Fix Ventura D, Menzel R (1992) The spectral input systems of hymenopteran insects and their receptor-based colour vision. J Comp Physiol A 170:23-40

Schaefer HM, Schaefer V, Levey DJ (2004) How plant-animal interactions signal new insights in communication. TREE 19(11):577-584

Schiestl FP (2005) On the success of a swindle: pollination by deception in orchids. Naturwissenschaften 92:255-264

Schiestl FP, Ayasse M, Paulus HF, Löfstedt C, Hansson BS, Ibarra F, Francke W (1999) Orchid pollination by sexual swindle. Nature 399:421-422

Spaethe J, Tautz J, Chittka L (2001) Visual constraints in foraging bumblebees: flower size and color affect search time and flight behavior. Proc Natl Acad Sci USA 98:3898-3903

Spaethe J, Moser WH, Paulus HF (2007) Increase of pollinator attraction by means of a visual signal in the sexually deceptive orchid, Ophrys heldreichii (Orchidaceae). Plant Syst Evol 264:31-40

Spaethe J, Streinzer M, Paulus HF (2010) Why sexually deceptive orchids have coloured flowers. CIB 3(2):1-3 
Streinzer M, Paulus HF, Spaethe J (2009) Floral colour signal increases short-range detectability of a sexually deceptive orchid to its bee pollinator. J Exp Biol 212:1365-1370

Uphoff W (1979) Anthocyanins in the flowers of European orchids. CMLS 35:1013-1014

van der Pijl L, Dodson CH (1966) Orchid flowers. Their pollination and evolution. University of Miami Press, Coral Gables
Vandewoestijne S, Róis AS, Caperta A, Baguette M, Tyteca D (2009) Effects of individual and population parameters on reproductive success in three sexually deceptive orchid species. Plant Biol 11:454-463

Vereecken NJ, Schiestl FP (2009) On the roles of colour and scent in a specialized floral mimicry system. Ann Bot 104(6):1077-1084 